\title{
Studying the Infrared Spectroscopy of the Pure Aluminum Oxide and Europium Doped Aluminum Oxide Compounds
}

\author{
Ahmad Khoudro $^{1} \quad$ Talal khalas $^{2} \quad$ Abd almajeed Krawe ${ }^{3 *}$ \\ 1.Professor, Department of Physics, Faculty of science, Tishreen University, Syria. \\ 2.Assistant Professor, Department of Physics, Faculty of science, Tishreen University, Syria \\ 3.MSc Student, Department of Physics, Faculty of science, Tishreen University, Syria
}

\begin{abstract}
The study of transparent oxides (TCO) is of great scientific importance due to its wide scientific applications. Therefore, our study came to some of the physical properties of the compounds of both pure aluminum oxide and europium doped aluminum oxide. By measuring the infrared spectrum of pure aluminum oxide, it is found that there are five vibratory frequencies, namely:

$(3523-3457-2450-1647-719) \mathrm{cm}^{-1}$ and five vibratory frequencies for europium doped aluminum oxide at $0.003 \%$ :

$(3518-3435-3175-2385-1580) \mathrm{cm}^{-1}$ The study showed that the absorbance values, the absorption coefficient, the refractive index and the optical length were in the europium doped sample by $0.003 \%$ greater than that of the pure aluminum oxide compound and are respectively: $\alpha=34.96 \mathrm{~cm}^{-1} \mathrm{n}=5.68$.

Keywords: aluminum oxide - Infrared spectrum - absorption coefficient - refractive index - Optical conductivity
\end{abstract} DOI: $10.7176 / \mathrm{CMR} / 10-5-01$

Publication date:May $31^{\text {st }} 2020$

\section{Introduction:}

The technology of semiconductors plays a very important role in our daily life due to its distinctive properties that made it one of the most important materials. Among the most important semiconductors, we mention in particular Transparent conductive oxides that attracted the attention of many researchers through their contribution to the development of several research and industrial fields, including the applications of optoelectronics and solar batteries [1-2].

Studies based on semiconducting materials rely heavily on transparent conductive oxides, thanks to its distinctive characteristics in addition to its low cost. Aluminum oxide is among the transparent oxides that have received great importance among researchers, especially in recent years, and this is thanks to its dual properties for it has high transparency in the visual field in addition to the advantage of having good electric conductivity [3].

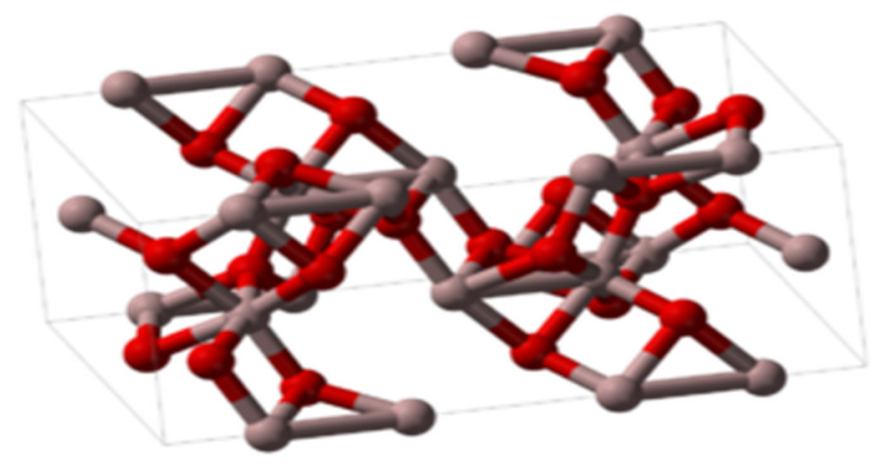

Figure 1: The crystalline structure of pure aluminum oxide

Aluminum oxide is a chemical compound with the formula $\mathrm{Al}_{2} \mathrm{O}_{3}$ (Figure 1) and it is also called as alumina. It exists in two patterns that differ from each other in the crystalline structure, and therefore differ in the physical and chemical properties in addition to applications.

Aluminum oxide is formed from heating aluminum hydroxide to about $400^{\circ}$, so we get a fine white powder that is greedy for moisture. It dissolves in all acids and it is used in chromatography, in drying processes, and in removing the colors of solutions.

\section{Infrared spectroscopy:}

Infrared is electromagnetic waves and it has all the basic properties of light, which are represented by the phenomena of diffusion, reflection, refraction, interference, diffraction and polarization. They are invisible thermal waves emitted by the sun or from artificial sources and have a high penetration ability as well as from our bodies and their frequency is lower than the red ray frequency in the visible electromagnetic spectrum. The infrared 
spectrum is located between the visible spectrum and the microwave radiation spectrum. It is divided into three zones, as follows:

- $\quad$ - Near infrared (NIR): It is the closest to the visible rays, namely the red color, and it lies within the range $[4000-12000] \mathrm{Cm}-1$

- $\quad$ - Middle Infrared MIR: located between the two preceding zones within the range [200 - 4000] $\mathrm{Cm}^{-1}$

Infrared spectroscopy is one of the basic methods of studying materials. It enables us to identify the structure of the material without affecting its properties. It depends on the study of the spectra absorbed by the sample, and its field is limited to $[20-1400] \mathrm{Cm}^{-1}$

Red radiation energy is not enough to cause electronic excitation in most materials, but it is sufficient to cause elasticity vibrations and flexion in the bonds. All types of these bonds respond to this amount of energy in which vibrations of this type occur. Therefore, they are absorbed in the zone beneath the red under the condition that absorption leads to a change in the polar moment, and these vibrations are quantized, and their occurrence means that the compound absorbs infrared energy in a specific part of the spectrum. [9]

Most spectroscopic analysis occur in the central infrared zone $[20-1400] \mathrm{Cm}^{-1}$ where the most molecular vibrations occur to determine the molecular structure of the studied compounds.

\section{Infrared spectroscopy principle}

Natural molecules vibrate according to all their vibrating patterns, but with very weak amplitudes. However, the photon has a sinusoidal electric component. If the frequency of the photon corresponds to the frequency of the vibrations of the normal patterns of the molecule, the molecule will enter the resonance and vibrate at very large capacities. In other words the photon whose energy is Equal to the energy necessary for the molecule to pass from a low energy state to an excited state is absorbed and its energy is transformed into a vibration energy as in Figure (2).

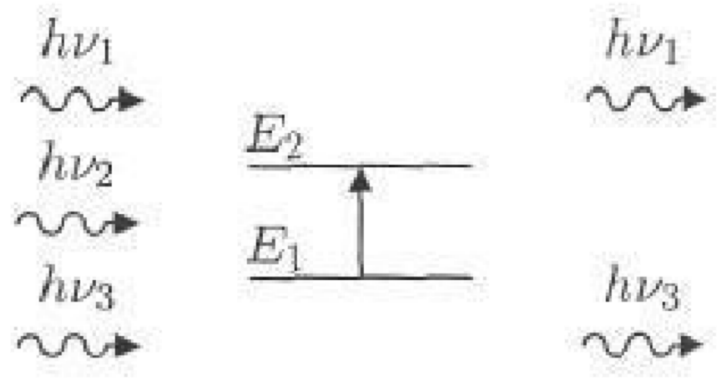

Figure (2) Infrared absorption

Only the photon whose energy (hv) equals to the transmission energy (E2-E1) is absorbed and thus the emission of the emitted radiation is impaired. As the absorption of some of the incoming photons leads to the appearance of the lines of compatibility of the photons that were not emitted in the curve of the infrared spectrum of the molecule. This absorption distinguishes the bonds between the atoms, since each vibration pattern corresponds to the single movement of the molecule, so there is a direct correspondence between the frequency of the absorbed radiation and the structure of the molecule [10].

\section{Research objective:}

This work aims to determine the field of absorption frequencies, that is, the vibrations samples frequencies of the infrared spectra of pure aluminum oxide and europium doped aluminum oxide by $0.003 \%$, and then finding the absorbance, absorption coefficient, damping factor, refractive index, optical length and optical conductivity to improve the physical properties of aluminum oxide.

\section{Research materials and methods:}

The following materials have been used in preparing the samples:

- $\quad$ Aluminum oxide $\mathrm{Al}_{2} \mathrm{O}_{3}$ (99\% purity, TITAN BIOTECH LTD, origin India)

- $\quad$ Europium $A u$ (purity 99\%, Amazon online marketing website, origin Italy)

\section{Devices and tools used:}

1- Sensitive scale type (SARTORIUS) with an accuracy of $\left(10^{-4}\right) \mathrm{gr}$ is available in the Faculty of Science Physics Department.

2- Small agate mortar.

3- High temperature thermal Oven $\left(1200^{\circ} \mathrm{C}\right)$ with a Temperature Regulator. 


\section{Preparing the samples:}

The samples are prepared by the solid state reaction method. Accordingly the weights of the powders required for each sample are mixed and calculated using the molecular weight method in order to obtain the compounds required for the study where $\mathrm{Al}_{1-\mathrm{x}} \mathrm{Au}_{\mathrm{x}} \mathrm{O}_{3} ;(\mathrm{x}=0.0-0.003)$. Then grinding these materials in the agate mortar perfectly well to make the mixture homogeneous and sifting it with a sieve of $90 \mu \mathrm{m}$. Then it is put it in a container and we add distilled water to increase the mixing process and homogeneity of the powder. Then we put it on a heater for 3 hours at a temperature of $100^{\circ} \mathrm{C}$ and the mixing and homogeneity process of the powder occurs by stirring.

After that, the powder is placed on a heater with direct contact with the air, then the water evaporates and then we perform a preliminary roasting process inside the oven (pre-sinter) to increase the degree of homogeneity of the mixture. We fix the oven temperature at $700^{\circ} \mathrm{C}$ for three hours, then we turn off the oven, which means to stop the roasting process and leave the sample inside the oven until it cools and reaches room temperature, thus we get rid of impurities that evaporate at high temperatures.

Then we grind the powder resulting from the roasting process in its first stage. Then we perform the second roasting process where we fix the oven temperature at $100^{\circ} \mathrm{C}$ for an hour and then we raise the temperature $50^{\circ} \mathrm{C}$ every $15 \mathrm{~min}$ until we reach the temperature of $700^{\circ} \mathrm{C}$ where we fix the oven temperature at it for 3 hours In order to get the crystal structure in its correct form.

To study the infrared spectra, we use an infrared spectroscopy device, which is a simple device whose main components are an infrared source, a sample holder and a detector. This device is considered one of the best spectroscopic devices used to identify the chemical composition of the compounds. It is available in the Faculty of Science - Tishreen University works at the range [400-4000] $\mathrm{Cm}^{-1}$.

The spectrometer is characterized by a computer memory that analyzes the waves gathered on the detector, computerizes them, and draws the spectrum resulting from absorption. Or a vibratory transmission of the atoms occurs relative to each other in the molecule, which leads to a periodic change in the length of chemical bonds or a change in the angles between the chemical bonds in the molecule. Each vibrational motion results from the movement of two atoms, or it may include a group of its constituent atoms. The wavelength or frequency at which this absorption occurs depends on several factors, including the mass of the atom, the strength of the bonds that make up the molecule, and the geometry of the atoms in the molecule.

\section{Results and discussion:}

The IR spectrum of pure aluminum oxide and europium doped aluminum oxide was measured using the spectrometer asco type FT / IR-460 plus available in the central laboratory of the Faculty of Science - Tishreen University, working in the range [400-4000] $\mathrm{Cm}^{-1}$. Where the permeability $\mathrm{T}$ was measured by the frequency function $v$, the absorbance $\mathrm{A}$, the absorption coefficient $\alpha$, the damping factor $\mathrm{K}$, the refractive index $\mathrm{n}$ and optical conductivity $\boldsymbol{\sigma}_{\mathrm{opt}}$ were calculated:

1- Permeability T: It is defined as the ratio between the intensity of the penetrating radiation to the intensity of the fallen radiation, it has been taken from the device itself and then by using the appropriate mathematical equations, other optical parameters have been calculated.

2- Absorbency A: is the ratio between the intensity of the absorbed radiation and the intensity of the fallen radiation, calculated from the equation [11]:

T represents Permeability.

$$
\mathrm{A}=\log \left(\frac{100}{T \%}\right)=\log \left(\frac{1}{T}\right)
$$

3- Absorption coefficient $\boldsymbol{\alpha}$ : defined as the ratio between the decrease in the flow of the fallen radiation energy to the unit of distance towards the spread of the fallen light wave within the field, and is calculated from the equation:

$$
\alpha=2.303 \frac{A}{d}
$$

A represents absorbency, $\quad \mathrm{d}=1 \mathrm{~mm} \quad$ the thickness of the material

4- The damping factor $\mathbf{k}$ : is defined as the amount of energy absorbed by the electrons of the studied material from the energy of the radiation photons that fall on it, and is calculated from the equation [13]:

$$
\mathrm{k}=\frac{\alpha}{4 \pi v}
$$

5- Refractive index $\mathbf{n}$ : which is the ratio between the speed of light in the vacuum to its speed in the field, and it is calculated from the equation [14]:

$$
\mathrm{n}=\left(\frac{100-T \%}{T \%}\right)^{1 / 2}
$$

6- Optical length $\mathbf{L}$ : the inversion of the absorption coefficient [12]:

$$
\mathrm{L}=\frac{1}{\alpha}
$$

7- Optical conductivity $\sigma_{\text {opt: }}$ optical conductivity is related to the refractive index and the damping factor according to the following equation [14]: 


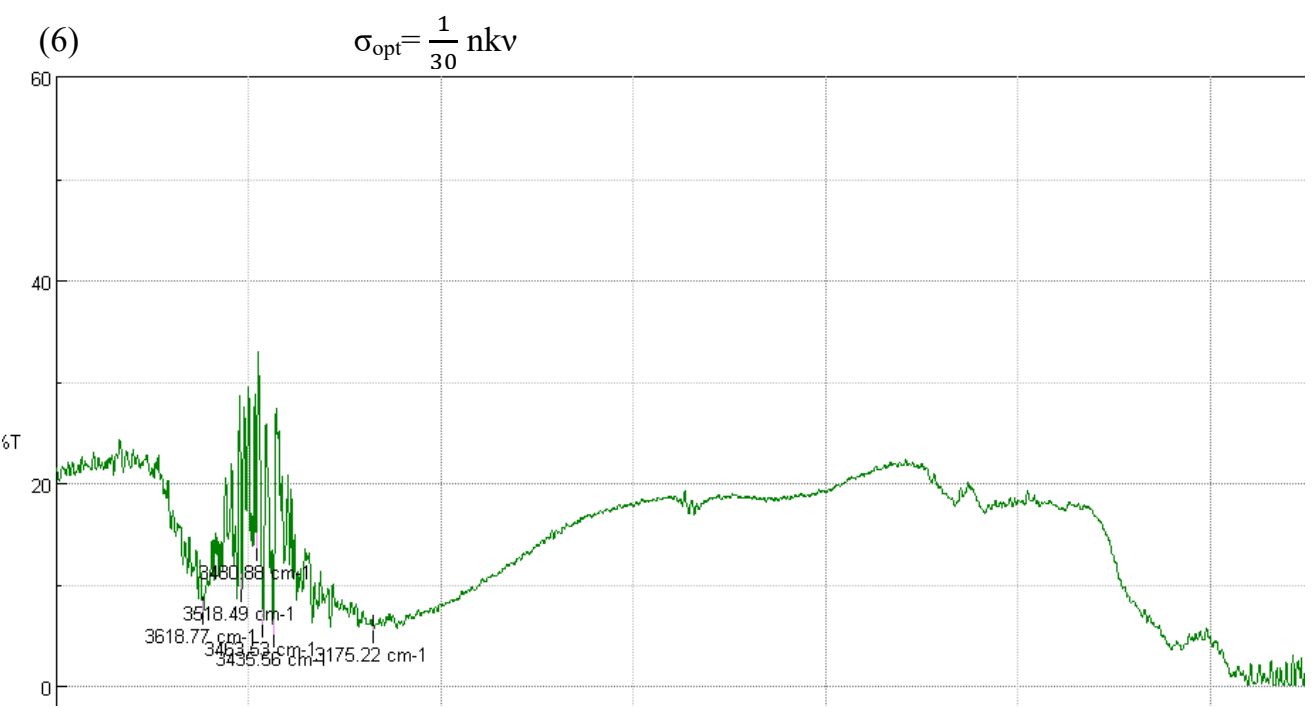

Figure (3): Represents the FTIR spectrum for pure aluminum oxide

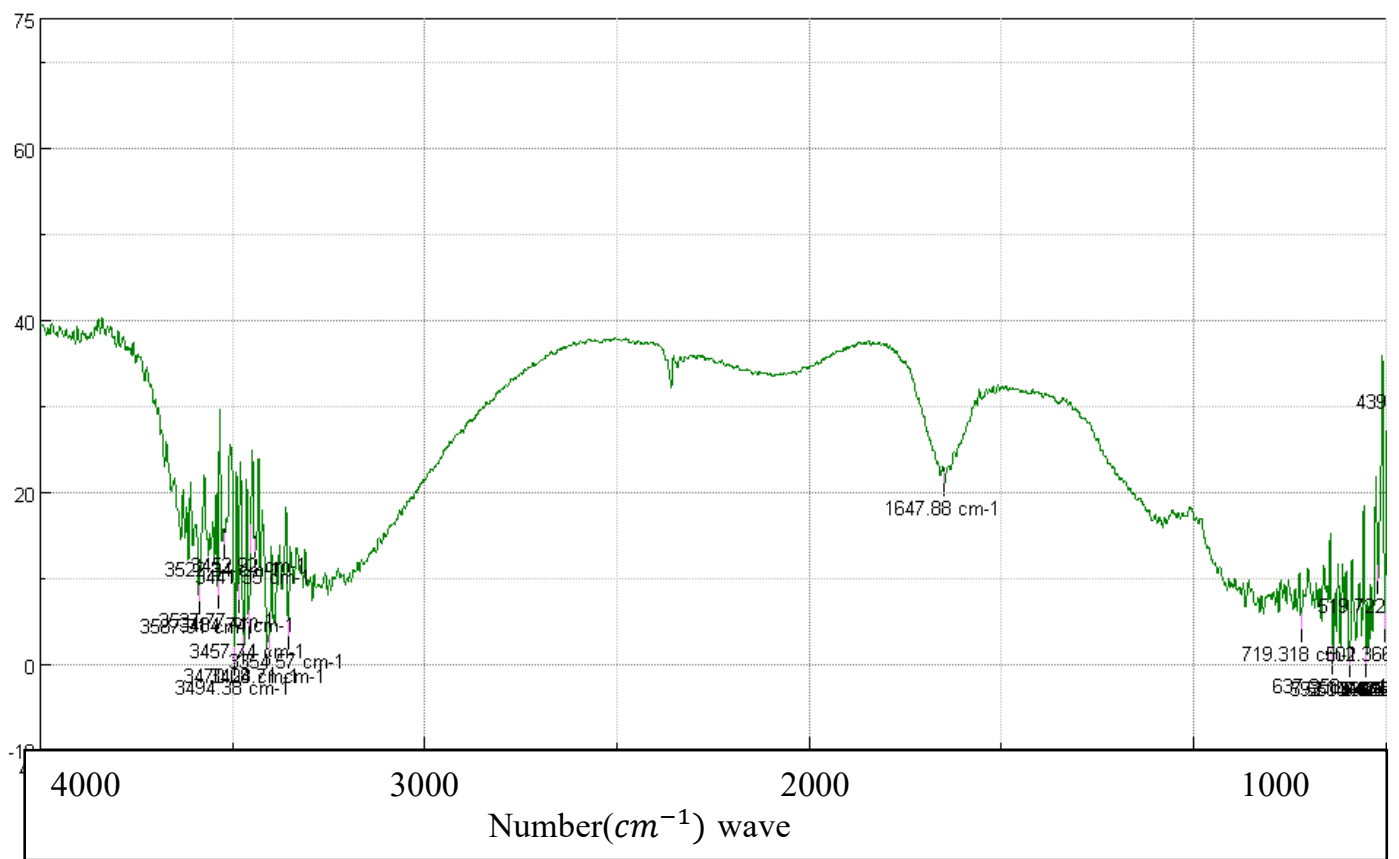

Figure (4) shows the FTIR spectrum of europium doped tin oxide by $(0.003 \%)$

FTIR analysis of samples has been performed at room temperature within the range [400-4000] $\mathrm{Cm}^{-1}$. Some bundles appeared within this range, including the absorption bundle at the top corresponding to the number of vibratory frequency $1630 \mathrm{~cm}^{-1}, 3518 \mathrm{~cm}^{-1}$ which belongs to the vibratory pattern of the hydroxyl group (vibration of hydroxyl group). This is due to the absorption of water vibration, the vibratory pattern also shows stretching and retraction (stretching vibrational mode) belonging to the $\mathrm{O}-\mathrm{H}$ group [15]. The location of the absorbent bundles and peaks also depends on the crystalline structure of the material and its chemical composition, as well as on the morphology of the material [16].

Pure aluminum oxide is characterized by a set of vibrational frequencies within the range [400-4000] $\mathrm{Cm}^{-1}$ which is: $(3523-3457-2450-1647-719) \mathrm{cm}^{-1}$

Europium doped aluminum oxide by $0.003 \%$ is characterized by a set of vibrational frequencies within the range $[400-4000] \mathrm{Cm}^{-1}$ which is:

$(3518-3435-3175-2385-1580) \mathrm{cm}^{-1}$

An absorbent value has been observed at about the frequency $3435 \mathrm{~cm}^{-1}$ belonging to the $\mathrm{C}-\mathrm{H}$ group, and this could be due to adsorption and interaction between carbon dioxide atoms with water during the sintering process [17].

From the permeability spectrum by the frequency function indicated in Figure (3), the physical quantities of 
pure aluminum oxide have been calculated, which are the absorbance, the absorption coefficient, the damping coefficient, the refractive index, the wavelength, the optical conductivity, which are shown in Table (1).

Table: (1) shows the vibrations frequency of pure aluminum oxide with corresponding permeability values for each frequency, absorbance and absorption coefficient, damping factor, refractive index, optical length and optical conductivity.

\begin{tabular}{|c|c|c|c|c|c|c|c|c|c|}
\hline$v$ & $\mathrm{~T} \%$ & $n^{2}$ & $A$ & $n$ & $\alpha\left(\mathrm{cm}^{-1)}\right)$ & $\mathrm{K} \times 10^{-4}$ & $\mathrm{~L}(\mathrm{~cm})$ & $\mathrm{nK} v$ & $\sigma_{\text {opt }}\left(\Omega c m^{-1}\right)$ \\
\hline 3523 & 11 & 8,06 & 0,95 & 2,84 & 21,85 & 4,93 & 0,045 & 4,93 & 0,16 \\
\hline 3457 & 2 & 43,56 & 1,69 & 6,60 & 38,87 & 0,89 & 0,025 & 2,03 & 0,06 \\
\hline 2450 & 32 & 24,60 & 0,49 & 4,96 & 11,27 & 3,66 & 0,088 & 4,44 & 0,14 \\
\hline 1647 & 22 & 3,53 & 0,65 & 1,88 & 14,95 & 7,22 & 0,066 & 2,23 & 0,07 \\
\hline 719 & 1 & 98,80 & 2 & 9,94 & 46 & 0,50 & 0,021 & 0,35 & 0,01 \\
\hline
\end{tabular}

The absorption coefficients variations are drawn by the function of the vibrational frequency of pure aluminum oxide as shown in Figure (5).

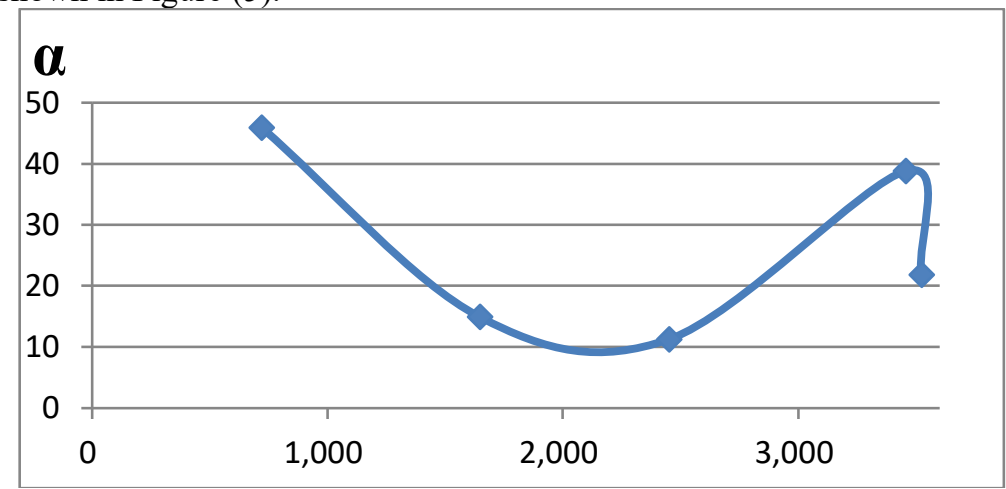

Figure (5) shows the absorption coefficient variations by the function of the vibrational frequency of pure aluminum oxide

We noticed that the largest absorption coefficient value was $46 \mathrm{~cm}^{-1}$ corresponding to the frequency 719 and the smallest absorption coefficient value was $11.27 \mathrm{~cm}^{-1}$ corresponding to the frequency 2450 .

The optical conductivity variations are also drawn by the frequency function of pure aluminum oxide, as shown in Figure (6).

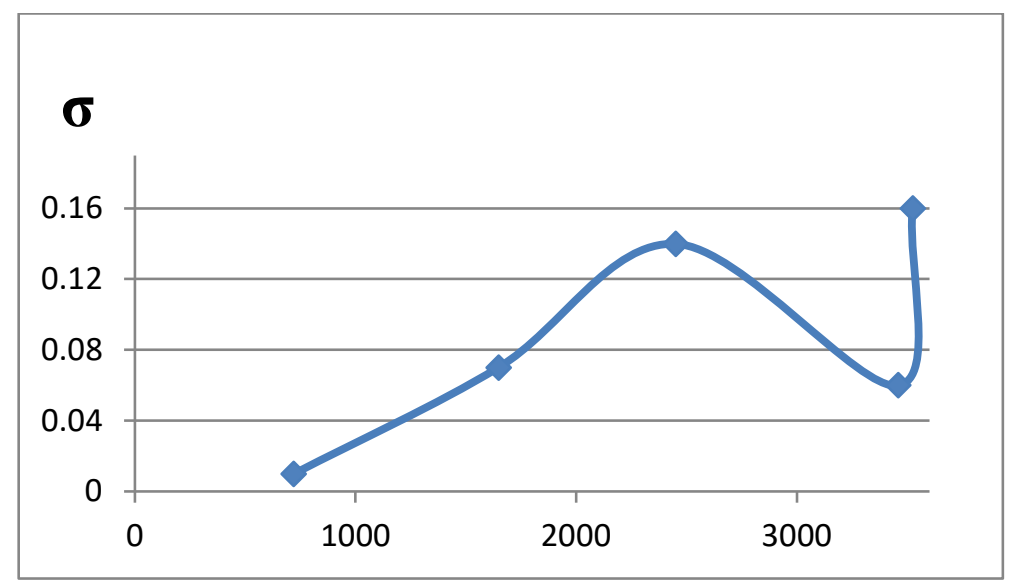

Figure (6) shows the studying of the conductivity variations by the frequency function of pure aluminum oxide.

We noticed that the largest value of the conductivity was $0.16\left(\Omega \mathrm{cm}^{-1}\right)$ corresponding to the frequency 3523 and the smallest value of conductivity was $0.01\left(\Omega \mathrm{cm}^{-1}\right)$ corresponding to the frequency 719 


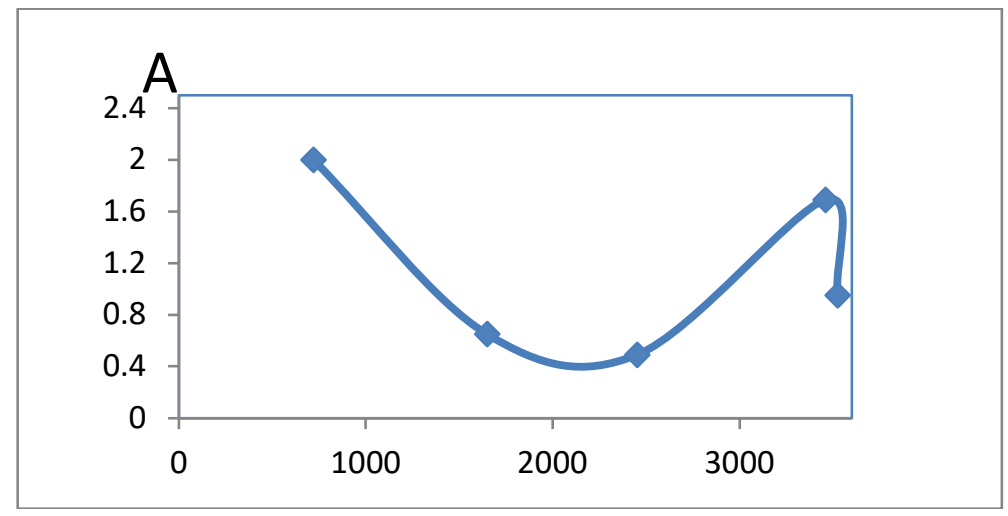

Figure 7: Absorbance variations by the frequency function of pure aluminum oxide powder

Absorption variations have been studied by the frequency function as in Figure (7). We note that the absorbance in the pure sample ranges between [2 -0.49]. The largest absorbance value was 2 corresponding to the frequency 719 and the smallest value of absorption was 0.49 corresponding to the frequency 2450 .

After doping the aluminum oxide with the europium element by $(0.003 \%)$ then measuring the permeability spectrum $\mathrm{T}$ by the vibrational frequency function as in Figure (4) where the physical quantities of the europium doped aluminum oxide by $(0.003 \%)$ represented by the absorbance, absorption coefficient, damping factor, and the refractive index and the wavelength and the optical conductivity of the corresponding frequencies have been calculated. (2)

Table: (2) shows the vibrations frequency of the europium doped aluminum oxide by $(0.003 \%)$ with corresponding permeability values for each frequency, absorbance and absorption coefficient, damping factor, refractive index, optical length and optical conductivity.

\begin{tabular}{|c|l|c|c|c|l|l|l|l|l|}
\hline$v$ & $\mathrm{~T} \%$ & $n^{2}$ & $A$ & $n$ & $\sigma\left(\mathrm{cm}^{-1)}\right)$ & $\mathrm{K} \times 10^{-4}$ & $\mathrm{~L}(\mathrm{~cm})$ & $\mathrm{nK} v$ & $\sigma_{\text {opt }}\left(\Omega c m^{-1}\right)$ \\
\hline 3518 & 8 & 11,5 & 1,09 & 3,39 & 25,07 & 5,22 & 0,039 & 6,22 & 0,20 \\
\hline 3435 & 3 & 32,3 & 1,52 & 5,68 & 34,96 & 8,09 & 0,028 & 15,78 & 0,52 \\
\hline 3175 & 4 & 23,9 & 1,39 & 4,89 & 31,97 & 8,01 & 0,031 & 12,43 & 0,41 \\
\hline 2385 & 17 & 4,88 & 0,76 & 2,20 & 17,48 & 5,83 & 0,057 & 3,05 & 0,10 \\
\hline 1580 & 16 & 5,25 & 0,79 & 2,29 & 18,17 & 9,15 & 0,055 & 3,31 & 0,11 \\
\hline
\end{tabular}

Absorption variations have been studied by the frequency function of the powder of the europium doped aluminum oxide, explained in (Figure 8) where we note that the absorbance in the pure sample ranges between [1.52-0.76] that the largest absorbance value was 1.52 corresponding to the frequency 3518 and the smallest value of absorbance was 0.76 corresponding to the frequency 2385 .

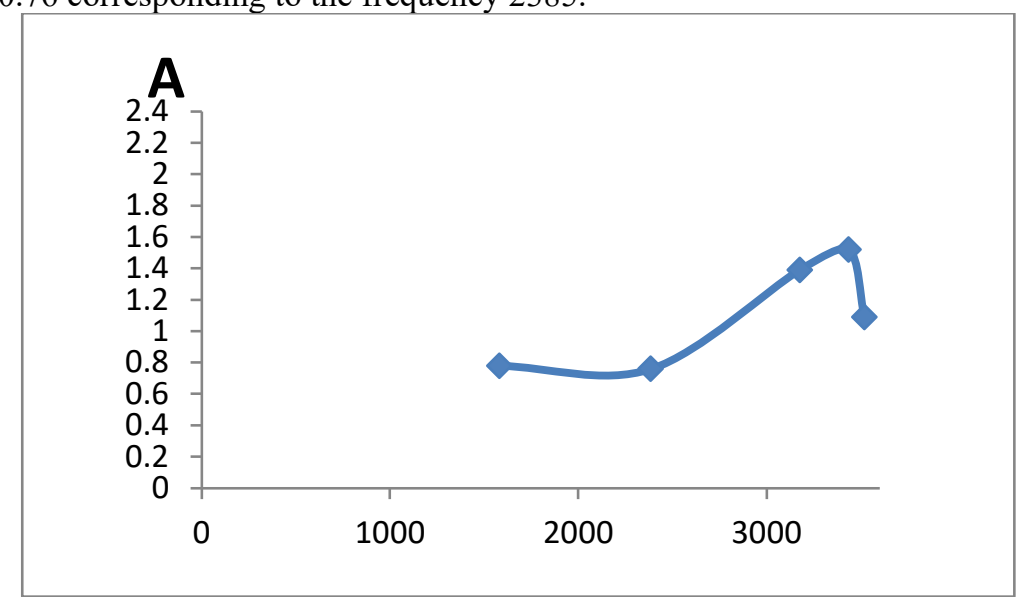

Figure (8) Absorption variations by the frequency function of the powder of the europium doped aluminum oxide

Figure (9) shows the variations of the conductivity $\sigma_{\text {opt }}$ by the frequency function of the doped sample and that the largest value of the conductivity was $0.52\left(\Omega \mathrm{cm}^{-1}\right)$ corresponding to the frequency 3435 and the smallest value of the conductivity was $0.1\left(\Omega \mathrm{cm}^{-1}\right)$ corresponding to the frequency 2385 


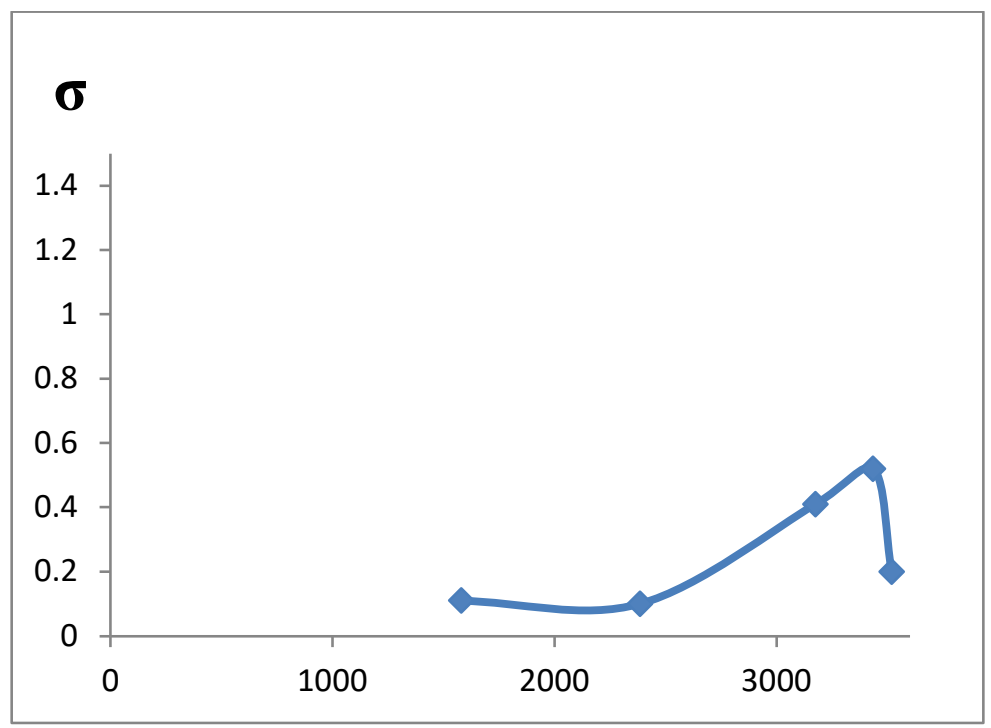

Figure (9) shows the variations of the conductivity $\sigma_{o p t}$ by the frequency function of the europium doped aluminum oxide

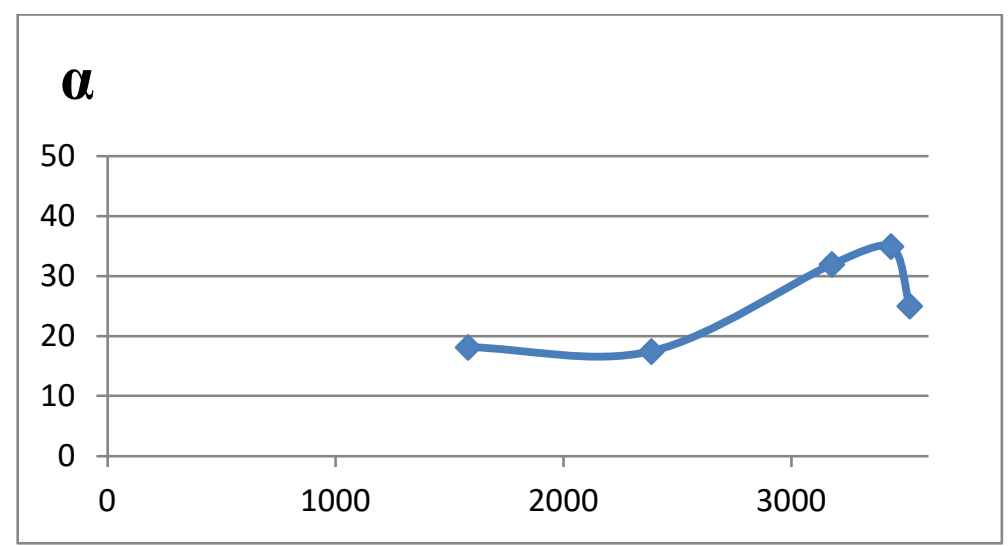

Figure (10) shows the variations of the absorption coefficient by the frequency function of the europium doped aluminum oxide

The absorption coefficient variations have been studied by the frequency function as in Figure (10), and we noticed that the largest value of Toheen coefficient was $34.96 \mathrm{~cm}^{-1}$ corresponding to the frequency 3435 and the smallest value of Toheen coefficient was $17.48 \mathrm{~cm}^{-1}$ corresponding to the frequency 2385 .

\section{Conclusions}

1. The pure aluminum oxide FTIR spectrum has shown some vibrational frequencies within the range [4004000] $\mathrm{Cm}^{-1}$ which are: (3523-3457- 2450-1647-719) $\mathrm{Cm}^{-1}$

2. The FTIR spectrum of the europium doped aluminum oxide by $0.003 \%$ showed vibrational frequencies within the range [400-4000] $\mathrm{Cm}^{-1}$, the most notably are: $(3523-3457-2450-1647-1120) \mathrm{cm}^{-1}$

3. 4. The absorbance value for the pure sample varies within the range [0.49-2], and for the doped sample, the absorbance value varies within the range [0.74 -1.52].

4. The absorption coefficient value varies within the range of the pure sample [46 - 11.27] $\mathrm{Cm}^{-1}$

5. The value of the refractive index for the pure sample varies within the range [9.94-1.88].

6. The value of the optical length $L$ concerning the pure sample varies in the range $[0.021-0,088] \mathrm{cm}$, and for the doped sample the value of the optical length varies within the range $[0.028-0.058] \mathrm{cm}$

7. The optical conductivity value $\sigma_{\mathrm{opt}}$ for the pure sample varies within the range $[0.001-0.16](\Omega \mathrm{cm})^{-1}$, for the doped sample, the value of the optical conductivity varies in the range $[0.11-0.52](\Omega \mathrm{cm})^{-1}$

8. The largest values of absorbance, absorption coefficient, refractive index and optical length were greater in the europium-doped sample by $0.003 \%$ for the vibrational frequency $3435 \mathrm{~cm}^{-1}$

9. The largest values of the absorbance, the refractive index, and the absorption coefficient were in the pure sample for the frequency $719 \mathrm{~cm}^{-1}$ 


\section{Reference}

[1]- SICILIANO.P, 2000,Preparation characterization and applications of thin films for gas sensors prepared by cheap chemical method Sensors and ActuatorsB:chemical, Vol. 70, N.1,p153-164.

[2]- BURST. J. M, 2010,Permittivity-Engineered TransparentConducting Tin Oxide Thin Films: From Deposition To Photovoltaic Applications, these de doctorate.

[3]- GORDON.Roy G,2000, Criteria for Choosing Transparent Conductors, MRS Bulletin, Vol. 25, N.8, p52-57.

[4]- THANGARAJOU.B,2002, Structural and electrical studies on highly conducting spray deposited fluorine and antimony doped $\mathrm{SnO} 2$ thin films from $\mathrm{SnCl} 2$ precursor, Thin Solid Films, Vol. 402, $\mathrm{N}^{\circ} .1, \mathrm{pp} .71-78$.

[5]- ELONGOVAN.E, 2003, RAMAMURTHI.K, Optoelectronic properties of spray deposited $\mathrm{SnO}_{2}$ : F thin films for window materials in solar cells, Journal of Optoelectronics and Advanced Materials, Vol. 5, $\mathrm{N}^{\circ} .1$, p 4554.

[6]- TURGUT.G,Et al.,2013,Effect of Nb Doping on Structural, Electrical and Optical Properties of Spray Deposited $\mathrm{SnO}_{2}$ Thin Films, Superlattices and Microstructures, Vol. 56, pp. 107-116.

[7]- MENOUER.K.L,2011, Etude et réalisationd'une cellule solairemulticouches du type $\mathrm{Si}_{-} \mathrm{SiO}_{2}-\mathrm{SnO}_{2}-\mathrm{ZnO}$ par APCVD, thèse de doctorat, UniversitéMouloudMammeri de TIZIOUZOU.

[8]- NISHINO.J, NOSAKA.Y,2004, Low température preparation of ZnO by a nearby vaporizing chemical vapor deposition method, Journal of Crystal Growth, Vol.268.

[9]- ROUESSAC.F, ROUESSAC,A,2004, AnalyseCHimiqueMéthodes et Techniques Instrumentals Modernes, Dunod, Paris.

[10]- MAGET.V,2005, Développement de Méthodes de traitement de signauxspectroscopiques: estimation de linge de base et du spectre de raie, Univ Henri Poicré.

[11]- SAKNI.L ,2017, Studying the structural of Fe doped tin oxide , Master thesis , Alwadi university.

[12]- ALJAWAD,S. et al 2016, studying effect of doping on the structural and optical properties of tin oxide thin films , journal of engineering and technology, Vol.34.

[13]- Mansour.M, 2012, studying the structural and optical properties of $\mathrm{ZnO}: \mathrm{Cu}$ thin films by APCVD method, Vol.5.

[14]- Zaid.A , 2012 ,studying the structural and optical properties of NiO thin films ,Master thesis, Dyala university, Iraq.

[15]- FAISAL.M, et al,2015, $\mathrm{SnO}_{2}$ doped $\mathrm{ZnO}$ nanostructures for highly efficient photocatalyst,Journal of Molecular catalysisA:chemical,Vol.39,pp.19-25.

[16]-SALEH.S.A,Et al, 2016,Structural and Optical Propertiesof Nanostructured Fe-Doped SnO2,Acta Physica Polonica A,Vol.129,PP.1220-1225.

[17]- GNANAM.S, RAJENDRAN.V,2010, Preparation of Cd-doped $\mathrm{SnO}_{2}$ nanoparticles by sol-gel route and their optical properties, Journal of Sol-Gel Science and Technology, Vol.56, pp. 128-133.

[18]- Sharma.A,et al.,2012, Magnetic Properties of Fe and $\mathrm{Ni}$ Doped $\mathrm{SnO}_{2}$ Nanoparticles, Nanomaterials and Nanotechnology, vol.1.

[19]- Mohamed. S.H.,et al.,2012,SnO2 dendrites-nanowires for optoelectronic and gas sensing applications, vol. 510, pp119-124.

[20]-SRINIVAS.K,2011,Structural, electronic and magnetic properties of $\mathrm{Sn}_{0.95} \mathrm{Ni}_{0.05} \mathrm{O}_{2}$ nanorods, Nanoscale, Vol.3, pp.642-653. 